\title{
MobSched: Customizable Scheduler for Mobile Cloud Computing
}

\author{
Suraj Sindia \\ Electrical and \\ Computer Engineering \\ Auburn University \\ Auburn, Alabama, 36849 \\ Email:szs0063@tigermail.auburn.edu \\ Alvin S. Lim \\ Computer Science and \\ Software Engineering \\ Auburn University \\ Auburn, Alabama, 36849 \\ Email: lim@eng.auburn.edu
}

\author{
Song Gao \\ Computer Science and \\ Software Engineering \\ Auburn University \\ Auburn, Alabama, 36849 \\ Email: song.gao@auburn.edu \\ Vishwani Agrawal \\ Electrical and \\ Computer Engineering \\ Auburn University \\ Auburn, Alabama, 36849 \\ Email: agrawvd@auburn.edu
}

\author{
Bobby Black \\ Computer Science and \\ Software Engineering \\ Auburn University \\ Auburn, Alabama, 36849 \\ Email: black85@tigermail.auburn.edu \\ Prathima Agrawal \\ Electrical and \\ Computer Engineering \\ Auburn University \\ Auburn, Alabama, 36849 \\ Email: agrawpr@auburn.edu
}

\begin{abstract}
In this paper, we explore how cloud computing techniques can be used on mobile devices. We analyze the reason why Hadoop's performance is poor in MANET, most notably, relying too much on distributed filesystem, and not aware of mobility and multi-hop nature of MANET. Two ways are proposed to deploy mobile cloud computing in an efficient manner: MobSched, a customizable job scheduler; and a mobile friendly MapReduce framework. These two methods enable developers to use MapReduce programming model in the context of MANET. Theoretical analysis suggests that the proposed framework can improve the performance of MapReduce jobs running on top of MANET, and reduce the energy consumption. Simulation results show that the proposed scheduler, MobSched, which is based on a linear programming formulation, can efficiently optimize multiple objectives such as power and (or) throughput, while being constrained with requirements such as minimum quality of service, and (or) maximum bandwidth usage that has to be met by the system. Comparison with other schedulers such as uniform load balancing, FIFO, and clustering types show that the proposed scheduler performs best when it comes to optimizing for a specific criteria such as total power consumption within reasonable latency.
\end{abstract}

\section{INTRODUCTION}

Personal mobile devices, especially smartphones and tablet computers, have been rapidly developed recently. Various technology advances make it possible to build powerful devices which are capable of handling much more complicated tasks that in a decade ago we did not expect even on a PC. In addition to computational capability advances, these mobile devices are also made small and light enough for everyday use. However, despite being powerful, in most of time hardware resource on mobile devices are not utilized. Smartphones are equipped with over $1 \mathrm{GHz} \mathrm{CPU}$ and powerful graphic processors, but they are kept idle or low utilized until the user plays games occasionally. Smartphones are equipped with advanced sensors such as accelerometer, GPS, etc. but the utilization is low. Smartphones have short distance wireless adapters that use the same standard protocols, but just stay silent from each other although their owners might be sitting right next to each other.

Hence, we believe it's promising to conduct cloud computing on mobile devices. It would allow a mobile node to delegate computing jobs to nearby nodes, thus improving resource utilization and potentially reduce job execution time. Consequently, we address a scheduling problem for mobile cloud computing networks that is aware of its operating conditions and factors this in to optimize the system performance.

It's been nearly 10 years since MapReduce programming model was introduced. However, most of current research on cloud computing is focused on big server clusters powered by high speed ethernet. Techniques and tools used on big server cluster cannot be directly applied to mobile clouds, due to various of limitations and attributes of mobile devices and mobile ad-hoc networks. This paper looks into parts where those techniques are not suitable in MANET, and aims at solving the challenges of mobile cloud computing.

In this paper, we locate the problems of mobile cloud computing, and propose a solution that tries to solve these problems. The solution we propose consists of a customizable scheduler that considers various factors on mobile devices, and a MapReduce framework that considers natures of wireless mobile ad-hoc network, and serves as an alternative to Hadoop.

The rest of this paper is organized as follows: Section II introduces the background of cloud computing and mobile ad-hoc network. Section III discusses related work in mobile cloud computing. Section IV gives a brief introduction on how Hadoop works, and analyzes why Hadoop performs poor in mobile ad-hoc networks. Section IV proposes a new MapRe- 
duce framework that is more friendly to mobile clouds than Hadoop. Section V proposes MobSched, a customizable job scheduler for mobile cloud computing. Section VI concludes this paper and describes our plan on future work.

\section{BACKGROUND}

\section{A. MapReduce}

MapReduce[4] is a programming model designed for processing parallelable problems. Users specify a map function that processes a key/value pair to generate a set of intermediate key/value pairs, and a reduce function that merges all intermediate values associated with the same intermediate key. Many real world tasks are expressible in this model. Programs written in this functional style are automatically parallelized and executed on a large cluster of commodity machines.

\section{B. Wireless Mobile Ad-hoc Network}

1) Mobility: Mobile devices are capable of being mobile. In vehicular networks, as an example, nodes are moving at 20 $\mathrm{m} / \mathrm{s}$ or higher speed. As a result, network topology can change from time to time. Assuming there's no crazy speeding cars, on high way the network topology may be relatively stable, but in an urban area, intersections creates a lot possibilities that vehicles can join or leave a group of other vehicles, making network topology change. When topology changes, the underlying transport protocol and network layer routing protocol need to handle it properly. In transport layer, for example, if TCP is used as transport protocol, there would be a period of disconnection when the sender would not receive any ACKs. The TCP protocol needs to differentiate between congestion and mobility caused disconnection. After the connectivity resumes, the protocol needs to resume TCP connection quickly as well. In network layer, the routing protocol also needs to be aware of the mobility. For example, frequent routing messages can help improve response time, but generates higher overhead.

Since network topology can change from time to time, and it's not easy to predict the change, mobility can also cause delay in computing. If a task is already assigned to a node, but before the node finishes the work, the node is disconnected from its corresponding nodes in the computation for a period of time, it can potentially block the whole computation at some point. If even worse, this node is disconnected too long, or completely shut down, the task assigned to it cannot be completed. Then other nodes have to be aware of this and re-assign the task to another node.

These issues caused by mobility make mobile devices significantly different from machines connected with wired networks which are used in traditional cloud computing. Mobility has to be considered and addressed properly when deploying cloud computing on mobile devices.

2) Bandwidth: Despite being developed significantly, wireless links are still slow. In [1], the authors present some empirical studies on IEEE 802.11n wireless adapter ad-hoc performance. As shown in Figure 1, even in best case 1-hop point-topoint scenario, the throughtput is only less than 24 Mbps. For
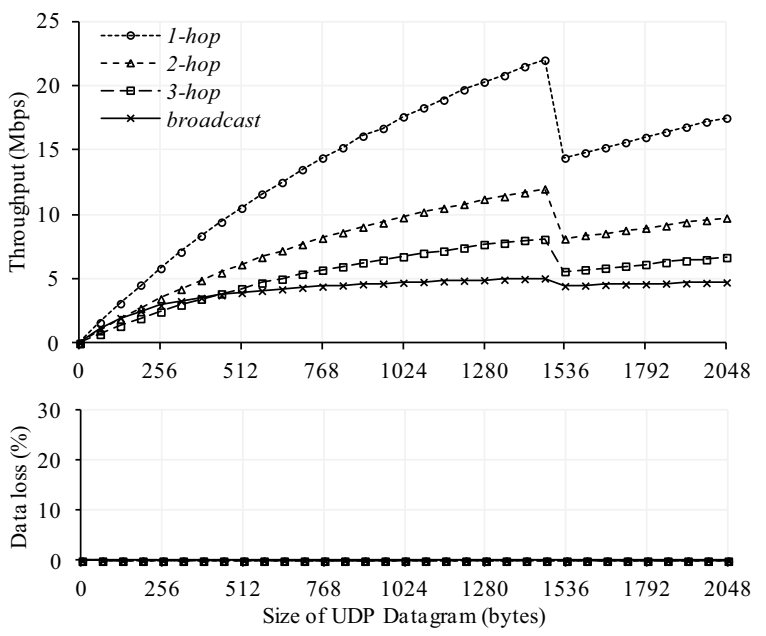

Fig. 1. Point-to-Point Performance on Ad-hoc network

multiple hops and situations where a lot of nodes are accessing the channel producing interference, the throughput becomes much worse. Compared to network devices used by traditional cloud computing, which are usually Gigabit Ethernet or even 100 Gigabit Ethernet, the bandwidth is significantly lower. Approaches used in traditional cloud computing that don't worry much about time used for transmitting data, need to be redesigned in order to adapt to mobile wireless networks, utilizing limited bandwidth more efficiently and minimizing issues such as interference that reduce the bandwidth.

3) Energy / Power Consumption: The need for mobile cloud computing-delegating resource intensive computation to remote servers, or other participating mobile nodes-is rooted in two important reasons:

1) Inadequate compute capability on the mobile node for a given application, or

2) Inadequate energy sources to power an application for a sustained amount of time.

It is therefore important to understand and model the power requirements of any given application before it can be justified for delegation to a cloud. Authors in [2] study the different tradeoffs involved in power/performance of a given application before it is justifiable for such an application to be delegated to the cloud. In a mobile cloud computing environment, the overall energy cost is equal to the sum of energies required for computation and communication. Therefore, authors in [2] propose a parametric model for finding energy savings in any cloud framework. The model considers the speeds of individual device and the remote cloud, number of instructions for computation, number of bytes transferred, network bandwidth available, and energy consumed by mobile device in idle, computing and communicating states. Their finding is that for maximum energy savings, offloading is the right choice when there is heavy computation but little communication.

4) Compatibility Among Different Mobile Devices: Another problem with mobile cloud computing is the variety of resources each node has as well the the operating system being 
used. There are currently over 9 mobile operating system including: iOS, Android, BlackBerry OS, S40, Symbian OS, Windows Phone, Bada, MeeGo, Tizen, and more being developed. [3] These run on hundreds of different mobile devices, each with their own different highly customized hardware. How will resources be allocated fairly among devices? Will a mobile device with a dual core 1 Gigahertz processor be used twenty times more than those devices with a single 100 Megahertz processor, or will they both only share a fraction of their processing. This concept can be applied to available memory and bandwidth as well. Some devices have 64 GigaBytes of flash memory, while others have 8 GigaBytes of memory. Newer devices have 4G capability, while older devices are stilled limited to $2 \mathrm{G}$ bandwidth. An incentive must be proposed that would encourage mobile owners to share their resources. This incentive could be a discount in cellular service, or priority speeds in mobile downloading or streaming.

\section{RELATED WORK}

\section{A. Hyrax: Porting Hadoop to Android}

Hyrax is a project that employs an approach of using MapReduce [4] framework to implement cloud computing on mobile devices. In the initial design [5], the author describes the goal of Hyrax project as "to develop a mobile-cloud infrastructure that will enable smartphone applications that are distributed both in terms of data and computation".

Hyrax uses Apache Hadoop [14], an open source MapReduce implementation in Java, in its platform. It targets the Android platform since it has Java VM which is required to run Hadoops Java code. In Hyrax, only worker nodes run on mobile devices. It still requires a PC server to run the master node.

In [5], a distributed multimedia search and sharing application is implemented to gain insight into the advantages of using Hyrax as an infrastructure. The experiments show that Hyrax easily scales to the 12 Android smartphones. However, it also exerts a huge base cost on Android, requiring a lot of time to process relatively small amounts of data. In their comparison experiments, servers are able to process 1000 5000 times more data than phones within the same amount of time.

[7] is a continuation of [5], aiming to make Hyrax more suitable for wide-scale deployment on the mobile devices of common users. New devices (Galaxy S) are used and a newer version of Hadoop (0.19.1) is ported.

It seems that authors of Hyrax did not address network scalability. Both versions of Hyrax use AP mode in the 802.11 wireless network. As a result, mobile devices are limited by the signal range of the master node, making it not scale as good as an ad-hoc network. The other thing we've noticed in the two Hyrax papers is that, nodes are placed very close to each other. In this case, all the nodes share the same wireless media, and interference can seriously affect network performance, hence may not reflect real application scenario properly.

\section{B. Misco}

In [8], a mobile MapReduce framework, Misco, is designed and implemented. Misco is targeted at any device that supports Python and network connectivity. In their experiments, Nokia N95 smartphones are used as testbed. Since the framework is implemented in Python, theoretically any mobile device running Python can work as a worker. An HTTP server is used for both user interacting and communication between master node and worker nodes. But for log server, UDP is used.

A set of experiments are conducted. The testbed consists of 30 Nokia N95 smartphones, which uses ARM CPU at 332 $\mathrm{MHz}$ and $90 \mathrm{MB}$ RAM, as worker nodes. The server is a commodity computer with $2 \mathrm{GHz}$ CPU and 640 RAM. The server connects to a $802.11 \mathrm{~g}$ router through $100 \mathrm{MBit}$ ethernet and all of smartphones are connected to the routing using 802.1g. Nokia Energy Profiler is used to take CPU, memory and power measurements. Results show that the overhead created by Misco is small. Misco software with data structures and Python libraries requires only 800KB of RAM. One of their interesting results is local task performance, which shows that, majority of processing is spent performing computations, and only a small fraction of the time is required for data transmission and cleanup, which is quite efficient for resource constrained devices.

However, the authors didn't compare with other framework or on other language. Since Python is an interpretive language, the performance can be pool for intensive computing. As a side effect, the portion of computing time in tasking time may not be able to reflect true network performance. In other words, if the same architecture is implemented on a compiled language, data transmission time may not use that little portion of tasking time, meaning Misco is not as efficient. This is left yet to be validated.

Similar to Hyrax, Misco also uses an AP mode 802.11 wireless network, which does not scale very well. From the figure, we notice it also places the nodes very close to each other.

\section{An Optical Character Recognition System}

Authors in [9] have explored leveraging an environment in which multiple mobile computers are available for computation. They experiment with an optical character recognition system that runs on multiple cell-phones. Such a system has a practical application in helping a traveler to capture foreign language characters (as an image) using the cell-phone camera, and subsequently running complex computation on multiple cell phones in the vicinity (for example those of other travelers) that are willing to share their computing resource to identify the foreign language characters that can finally be translated into the native tongue of the traveler.

\section{Mobile Friendly Framework For MapReduce}

\section{A. Problems of Hadoop in MANET}

Hadoop is designed for ethernet. Wireless networks invalidates many assumptions that hold true in ethernet. As revealed in Section III-A, studies in [5], [7] show that Hadoop running 
on mobile devices in 802.11 networks suffers from wireless interference and various factors, and has poor performance.

Hadoop closely relies on Hadoop Distributed File System (HDFS). Both input data and output data are handled by HDFS. However, using HDFS in 802.11 networks can be harmful in many cases. For example, HDFS uses pipeline between DataNodes to accelerate the replication process. However, in 802.11 networks, network $\mathrm{I} / \mathrm{O}$ instead of disk $\mathrm{I} / \mathrm{O}$ is the bottle neck, and pipeline brings heavy burden on the network. In addition, in mobile ad-hoc networks, nodes are not grouped into "racks" like in ethernet, as a result, the way HDFS uses to predict failure and handle recovery won't work. Since Hadoop uses HDFS as input of the computation, in some serious situation where failure rate is high, the computation would not be finished without re-loading input data into HDFS.

\section{B. Framework Design}

Aiming at solving Hadoop's problem in MANET, a mobilefriendly framework for MapReduce is proposed. In the proposed framework, there's no fixed topology. Master node can be any node in the MANET. We assume the using scenario in which any user can delegate a MapReduce job to other mobile nodes that are in the same Ad-Hoc network, in which case, the node delegating the job is the Master node for this particular job. A node can be Master node and worker node at the same time.

An important attribute of the proposed framework that makes it different from Hadoop is that, data is not pre-loaded onto any nodes in the cluster. Instead, the data is sliced into pieces as small as possible, and sent to worker nodes along with task descriptions. The tasks are small enough that they can be finished within reasonable time, so that even if the task fails completely, it doesn't cost too much time to re-execute it on another node. Like input data, the output results from Reduce functions are also sent directly to the Master node. ${ }^{1}$ Since there's no file system involved, we don't need to worry about the integrity of data. Data is always with tasks. If a task succeeds, the data produced by it is guaranteed to be delivered.

The details of the framework design will be described in another companion paper.

\section{MobSched: A Customizable Scheduler}

We propose a scheduler that can be optimized for multiple objectives such as power and (or) throughput, while constraining a minimum quality of service, and maximum bandwidth that has to be met by the system. In this section, we first formulate the scheduling problem, and then discuss its performance on three different metrics by comparing them against three other schedulers [10], namely: uniform, cluster, and FIFO.

\footnotetext{
${ }^{1}$ This assumes that the node delegating the job is the only one who needs the results. If other nodes need the results as well, there needs to be some mechanism to share the results with them, either through MANET, or by uploading to an Internet/Infrastructure-based cloud storage.
}

\section{A. Problem Formulation}

Consider the following scenario: Let there be $N$ mobile nodes in a closed network willing to mutually share their compute resources, let $p_{1}, \cdots, p_{N}$ be the power consumed by each node; let the bandwidth of the link between say the $i^{t h}$ node and rest of the network varies from $b_{1}, \cdots, b_{N}$, and the processor speeds of each node in the network varies from $f_{1}, \cdots, f_{N}$; so that the throughput guaranteed by each node to the $i^{t h}$ node becomes $t_{1}=b_{1} \times f_{1}, \cdots, t_{N}=b_{N} \times f_{N}$, and the error or link quality that can be guaranteed by each node to the $i^{t h}$ node vary from $e_{1}, \cdots, e_{N}$ then we set up the linear programming (LP) problem for minimizing the total power consumed and maximizing the system throughput, as shown in Eqn. 1-5 in Fig. 2, where $x_{1}, \cdots, x_{N}$ are the fraction of allocated work to each of the $N$ nodes, and is returned as the solution of this optimization problem. $\alpha$ and $\beta$ are the optimization parameters that weigh the relative importance of the two objectives, namely power consumption and system throughput, so that $\alpha, \beta$ should add up to 1 . This optimization problem assumes that the parameters such as link quality, link bandwidth, power consumption and processor speeds are available at each node to facilitate the optimization problem.

\section{B. Discussion}

The proposed scheduler, MobSched, was formulated as a LP problem in the previous section. Equation 1 represents a general form of the objective function used in MobSched, however, the objectives to be optimized by MobSched can be chosen as mandated by a specific application context. For example, in the Eqn. 1, we chose minimizing system power consumption, and maximizing throughput as our two objectives (while more than two objectives are allowed in MobSched), and constrained with the requirement of meeting a maximum tolerable error rate (or packet loss per unit time). The constraint in Eqn. 3 is from the need that all the fraction of a task assigned to a number of participating nodes must add up to unity. Finally, the constraint in Eqn. 4 helps bias the optimization problem towards achieving one of its objectives, for example, $\alpha=0.8, \beta=0.2$ lets the optimization put greater effort into minimizing system power as opposed to maximizing throughput.

To validate the LP problem set up earlier and compare its performance on different metrics against three other schedulers is attempted. We consider the problem of scheduling a big task, namely, optical character recognition of 1000 Chinese characters. We assume the resolution of individual task assignment to the nodes as 1 character. We assume 10 nodes including the host are at disposal to be engaged in computation. The optimization parameters $\alpha=0.8, \beta=0.2$ are chosen to deliberately bias maximizing system power over minimizing throughput. A maximum error rate $E=5$ is set as a constraint to be met. Typical values are chosen for $p_{1}, \cdots, p_{N}, e_{1}, \cdots, e_{N}$, and $t_{1}, \cdots t_{N}$. Figure 3 shows a comparison of normalized relative performance of the MobSched on three metrics, namely system power consumption, scheduler latency and error rate. Also shown are the metrics of 
Minimize :

$$
\alpha\left(p_{1} x_{1}+\cdots+p_{i} x_{i}+\cdots+p_{N} x_{N}\right)-\beta\left(t_{1} x_{1}+\cdots+t_{i} x_{i}+\cdots+t_{N} x_{N}\right)
$$

Subject to :

$$
\begin{gathered}
x_{1} e_{1}+\cdots+x_{N} e_{N} \leq E, \\
x_{1}+\cdots+x_{N}=1, \\
\alpha+\beta=1 \\
0 \leq \alpha, \beta \leq 1
\end{gathered}
$$

Fig. 2. MobSched optimization problem for distributed task scheduling. Refer Section V for a more detailed discussion on MobSched.

three other schedulers: uniform-schedules such that all nodes are uniformly loaded, cluster-multiple queues are allocated clusters, all jobs at a queue will have access to allocated cluster, and FIFO-Tasks are serviced in the order in which they are received on the first available resource. The Y-axis in Fig. 3 is shared by all three metrics, needing the axis to be normalized to enable relative comparison among the schedulers on the three metrics.

In Fig. 3, we see that MobSched performs better than the rest for the optimized parameter namely system power consumption at just under $9 \%$ on the relative scale, while the nearest rival is the uniform scheduler at $68 \%$. The scheduler latency- which is the time required to come up with this solution is a little over $56 \%$, but still acceptable considering the fastest scheduler FIFO has a latency of $33 \%$. The low latency of MobSched can be attributed to the LP problem that it solves internally, which is essentially a polynomial time solvable problem. Error rate constraint specified in the problem is also met by MobSched, and in the process gives one the least error rate among all four schedulers. This shows that by carefully choosing the constraints, one can achieve optimized solutions with MobSched at low latencies.

\section{CONCLUSiOn AND Future WORK}

In this paper, we analyze how cloud computing techniques can be used on mobile devices. Apache Hadoop is examined, and analyzed under the context of mobile ad-hoc networks. We speculate the reason why Hadoop's performance is poor in MANET, most notably, relying too much on distributed filesystem, and not aware of mobility and multi-hop nature of MANET.

We propose in this paper two ways to deploy mobile cloud computing in efficient way: MobSched, a customizable job scheduler, and a mobile friendly MapReduce framework. They enable developers to use MapReduce programming model, but respect to the context of MANET. Our theoretical analysis suggests that, the proposed framework can improve the performance of MapReduce jobs running on top of MANET, and reduce the energy consumption. Simulation results showed MobSched can optimize multiple objectives such as power and (or) throughput, while constrained with a minimum quality of service, and (or) maximum bandwidth that has to be met by the system, and can achieve these objectives at low latencies due to the inherent formulation of the scheduler as a polynomial time solvable LP problem.

In future work, we will implement the proposed scheduler and framework as real program. We will test them in an emulated environment, as well as real-world mobile devices.

\section{REFERENCES}

[1] Seungbae Lee, Alvin Lim, Reliability and performance of IEEE 802.11n for vehicle networks with multiple nodes, 2012 International Conference on Computing, Networking and Communications (ICNC)

[2] K. Kumar, Y.-H. Lu, Cloud computing for mobile users: can offloading computation save energy? Computer vol. 43, pp. 5156, 2010.

[3] Wikipedia, the free encyclopedia, Mobile Operating System. http://en. wikipedia.org/wiki/Mobile_operating_system, October 2012.

[4] Jeffrey Dean and Sanjay Ghemawat, MapReduce: Simplified Data Processing on Large Clusters. December, 2004.

[5] Eugene E. Marinelli, Hyrax: Cloud Computing on Mobile Devices using MapReduce. Carnegie Mellon University Tech. Report CMU-CS-09164, September 2009.

[6] Wikipedia, the free encyclopedia, Apache Hadoop. http://en.wikipedia. org/wiki/Apache_Hadoop, October, 2012

[7] Chye Liang Vincent Teo, Hyrax: Crowdsourcing Mobile Devices to Develop Proximity-Based Mobile Clouds. August 2012

[8] Adam Dou, Vana Kalogeraki, Dimitrios Gunopulos, Taneli Mielikainen, and Ville H. Tuulos, Misco: A MapReduce Framework for Mobile Systems. PETRA '10 Proceedings of the 3rd International Conference on PErvasive Technologies Related to Assistive Environments. 2010

[9] J. Cheng, R.K. Balan, M. Satyanarayanan, Exploiting rich mobile environments. Technical Report, 2005.

[10] F. Teng, Management of Data and Scheduling of Tasks on Distributed Architectures. Ph.D. Thesis, Ecole Centrale Paris, 2011.

[11] Robert Chansler, Hairong Kuang, Sanjay Radia, Konstantin Shvachko, and Suresh Srinivas: "The Hadoop Distributed File System." http://www. aosabook.org/en/hdfs.html

[12] Dhruba Borthakur: "HDFS Architecture Guide." http://hadoop.apache. org/docs/hdfs/current/hdfs_design.html

[13] Brad Hedlund: "Understanding Hadoop Clusters and the Network." http://bradhedlund.com/2011/09/10/ understanding-hadoop-clusters-and-the-network/

[14] Wikipedia: "Apache Hadoop." http://en.wikipedia.org/wiki/Apache_ Hadoop\#Hadoop_Distributed_File_System 


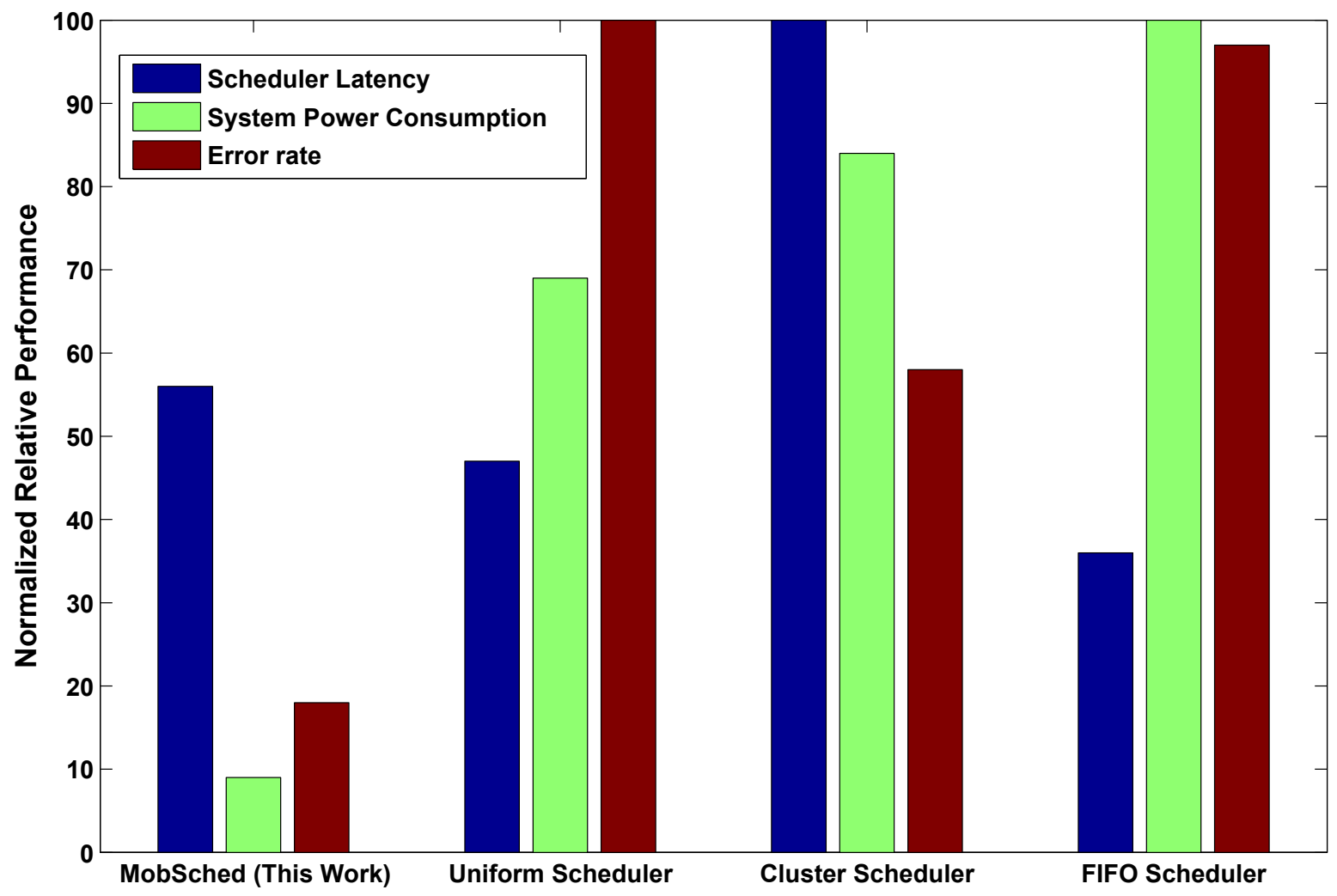

Fig. 3. Comparison of different performance metrics such as system power consumption, scheduler latency, and error rate of the proposed scheduler MobSched against three other schedulers. The common Y-axis in the graph is normalized by the maximum value of metric among the four schedulers.

[15] Eric Baldeschwleler: "Best Practices for Selecting Apache Hadoop Hardware." http://hortonworks.com/blog/ best-practices-for-selecting-apache-hadoop-hardware/
[16] Intel Corporation: "Hadoop* Clusters Built on 10GbE." http://www.intel.com/content/www/za/en/big-data/ 10gbe-10gbase-t-hadoop-clusters-paper.html 